\title{
Experimental Validation of FEA Modeling of Touch Trigger Probes
}

\author{
Mohd Rizal Salleh, Qing Ping Yang, Peng Wei, Barry Jones \\ School of Engineering and Design, Brunel University, Middlesex UB8 3PH, UK \\ Phone:+44 1895 265854, Fax:+44 1895 269763, Email: emstppy@brunel.ac.uk
}

Abstract -The authors have previously proposed the use of Finite Element Method (FEM) for the modeling of coordinate measuring machine probes. Whilst the modeling results have been published previously, this paper presents the detailed experimental validation to compare the FEM and experimental results. The comparison shows that the agreement is generally good with probing contacts at lower latitudes near the equator of the reference sphere. The differences between the modeling and experimental results become large at higher latitudes. This is believed to be mainly caused by the sliding effects which occur during probing contact in the experiments.

Keywords - Coordinate Measuring Machines (CMMs), Touch Trigger Probe, Pre-travel Variation, Finite Element Method (FEM), Model Validation

\section{INTRODUCTION}

Probe lobing and, in particular, probe pre-travel variations is the highly repeatable errors that have been studied by many researchers because of their great significance in CMM measurements $[1]-[8]$. They are usually characterized by the key influencing parameters or factors, e.g. probe stylus length, probing angle, probe orientation, probe stiffness, etc.

There are generally four methods used by various research groups. The early investigations were mainly performed by experiments where the relationships between the probe errors and the influencing variables are determined. This method is straightforward in principle, however, it is very time consuming due to the nature of 3D measurements. From the experimental data, neural network models can also be readily developed. Although neural network is very powerful for error prediction and correction, it experiences the same problem since large amounts of data are required to train the neural networks [2] - [3]. Another obvious approach is analytical mathematical models, which have been developed for some probes and their operations [4] - [6]. Analytical mathematical model is a simple, thus preferred modeling method, but it is limited to probes with simple structure.

The authors have proposed the use of finite element modeling (FEM) as a general approach to probe modeling [7]. The initial results and subsequent refinements have been reported in [8], together with the discussions of the applications of the FEM results, e.g. for the evaluation of probe measurement uncertainty. As theoretical models, FEM results have to be experimentally validated. Whilst the informal validation has been described in [8], this paper presents the detailed experimental validation. The comparison between modeling and experimental results under various conditions is presented.

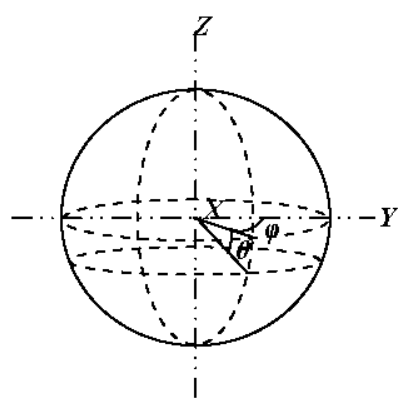

(a)



(b)

Fig. 1: (a) Probing angles and (b) Probe orientation

\section{PROPOSED METHODOLOGY}

In order to compare the modeling and experimental results, they should ideally be obtained from the same probe. Since our FEM was based upon a touch triggering probe, which had been mechanically disassembled, the experiments were performed with a different probe but of the same type and design. Further, the modeling and experiments were performed under the similar conditions with the same parameter values. The comparison mainly considers the influences of the key parameters, i.e. probe stylus length, approaching direction, probing latitude, probe orientation.

The experimental setup was based upon the measurement of a reference sphere, which simplifies the collection of the 
experimental data under the influences of the above parameters. The modeling was performed with the following configurations: three stylus lengths: $l=7 \mathrm{~mm}, 20 \mathrm{~mm}$ and 50 $\mathrm{mm}$, with the total length including stylus holder is $17 \mathrm{~mm}$, $30 \mathrm{~mm}$ and $60 \mathrm{~mm}$, respectively. The details of probe model have been explained in [7] and [8] including the dimensions and materials properties. However, for validation purpose, 20 $\mathrm{mm}$ and $60 \mathrm{~mm}$ styli were selected for experimental validation.

The influence of stylus length was studied together with those of probe orientations $A$ and probing angles, $\varphi$ and $\theta$, which representing longitude and latitude, respectively. They are defined in Fig. 1. $\theta$ has an interval of $15^{\circ}$, and $\varphi$ has an interval of $30^{\circ}$, and probe orientations $A$ at $0^{\circ}$ and $45^{\circ}$.

The contact force at different probing angles was generated from the mathematical modeling of probe in [4], which varies slightly at different probing angles. The error results generated from FEM are then directly compared with experim ental results to validate the modeling.

\section{Experimental Design}

The experiments were performed using a Renishaw TP2$5 \mathrm{~W}$ touch trigger probe with a Mitutoyo FN503 CMM. Due to their significant influences on the probe performance, the four parameters mentioned above were selected, i.e. stylus length $l$, probe orientation $A$, and approaching directions $\theta$ and $\varphi$.

In each experiment, six latitudes with 36 probing points at each latitude and one point at top of the reference ball with the total 217 points were measured, as shown in Fig. 2. The interval between points: $\varphi$, is $30^{\circ}$ and half of the reference sphere was equally divided by $\theta$ at $15^{\circ}$ between latitude. Each set of experiment used different combination of $\varphi, \theta, L$ and $A$. All measurements were repeated three times. Renishaw's probe head, $\mathrm{PH} 9$, was used in experiment to set the probe angle $A$. It was first set at vertical i.e. $A=0^{\circ}$, the equally distributed 217 points were measured at the six latitudes and at top of reference sphere. The probe body and stylus assembly was then rotated to $45^{\circ}$ and similar procedure was applied to measure half of reference sphere.

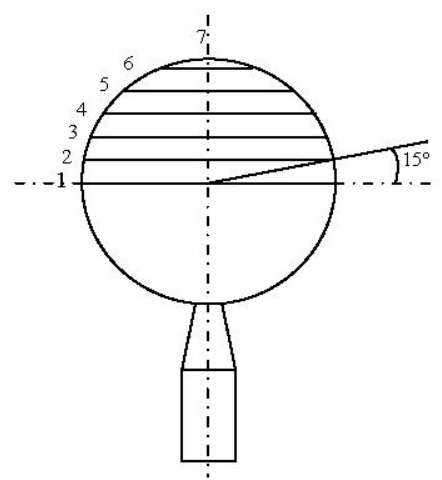

Fig. 2: Experimental sampling plan of $\theta$
Other probing characteristics were kept constant with a stylus diameter of $3 \mathrm{~mm}$, stylus tip diameter $4 \mathrm{~mm}$. Minimum trigger force was set to $7-8$ gf. Operating temperature was $20 \pm 2^{\circ} \mathrm{C}$ at $40 \%$ relative humidity. Reference ball diameter was $19.9960 \pm 0.0005 \mathrm{~mm}$.

Both FEA and experimental probe errors were computed using the measured coordinate points on the surface of reference sphere. The distance of a measured point relative to the centre of the reference ball is defined as $r_{i}$ :

$$
r_{i}=\sqrt{\left(x_{i}-x_{c}\right)^{2}+\left(y_{i}-y_{c}\right)^{2}+\left(z_{i}-z_{c}\right)^{2}}
$$

where $i=1,2,3, \ldots \ldots n ;\left(x_{c}, y_{c}, z_{c}\right)$ is the center of reference ball. The best fit least square circle, $R_{j}$, for $j=1,2, \ldots ., 6$ (latitude number) is obtained by averaging $r_{i}$, and hence

$$
R_{j}=\frac{1}{n} \sum_{i=1}^{n} r_{i}
$$

The pre-travel at ith latitude, $\delta_{i}$, becomes

$$
\delta_{i}=R_{j}-r_{i}
$$

\section{RESULTS}



Fig. 3: Comparison of the modeling and experimental error results (with $20 \mathrm{~mm}$ and $60 \mathrm{~mm}$ styli)

In general, the probe error patterns reflected the internal mechanical construction of the probe with three lobings corresponding to the tripod legs that support the stylus holder and stylus stem. Furthermore, stylus length significantly influences the size of probe lobing as shown in Fig.3. It is significantly increased and varied when longer styli were used. The lobing is proportional to stylus length. Fig. 3 shows that the agreement between experimental and FEA modeling results of $20 \mathrm{~mm}$ stylus was generally good with small differences. However, the differences become larger for $60 \mathrm{~mm}$ stylus. The standard deviations (SDs) of experimental and FEA results for $20 \mathrm{~mm}$ stylus are $3.05 \mu \mathrm{m}$ and $2.39 \mu \mathrm{m}$, respectively. The SDs for $60 \mathrm{~mm}$ stylus are 3.07 $\mu \mathrm{m}$ for experiment and $10.89 \mu \mathrm{m}$ for FEA modeling. 
Further validation was performed on $20 \mathrm{~mm}$ stylus to validate the model in accordance with probe orientation and probing direction.

Fig. 4 and 5 show the results of modeling and experiments when both were performed at $\mathrm{A}=0^{\circ}$ and $45^{\circ}$, respectively. It can be seen that the errors patterns were similar with a small $\theta\left(<30^{\circ}\right)$ and the differences becomes larger when $\theta>45^{\circ}$. They generally show how stylus configuration, contact points and approach directions influenced the probe lobing.

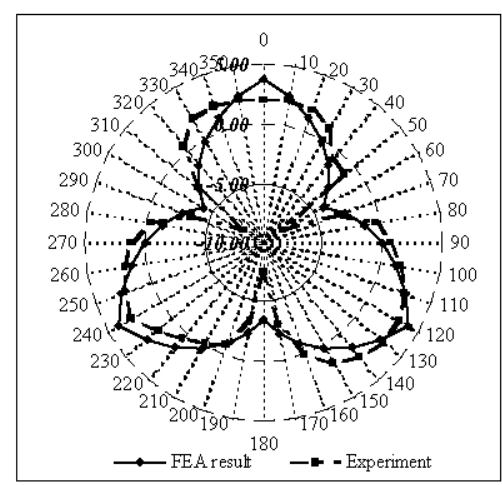

$\theta=0^{\circ}$



$\theta=45^{\circ}$

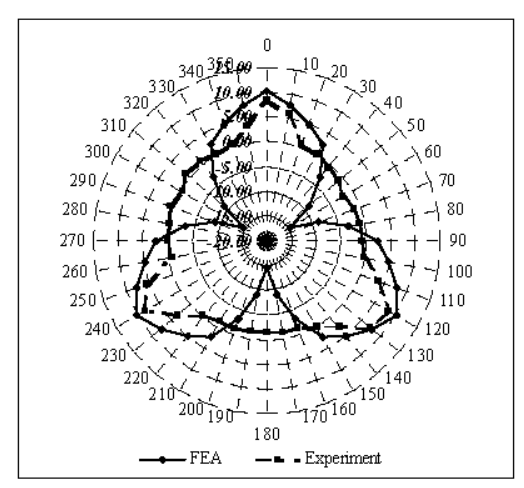

$\theta=15^{\circ}$

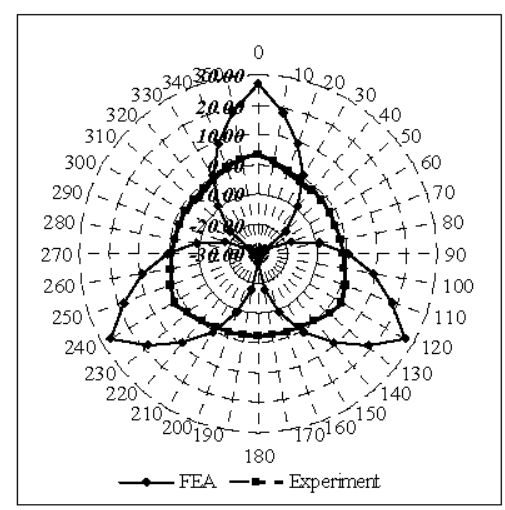

$\theta=60^{\circ}$

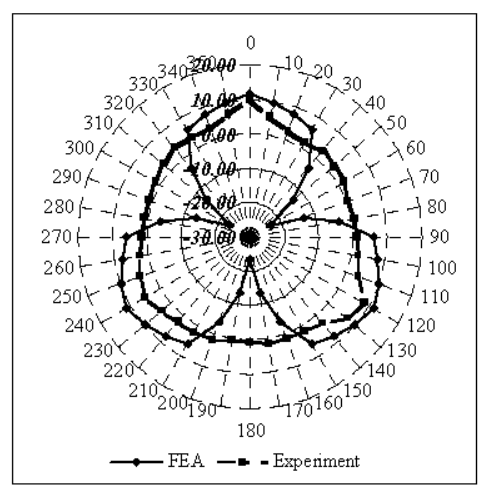

$\theta=30^{\circ}$

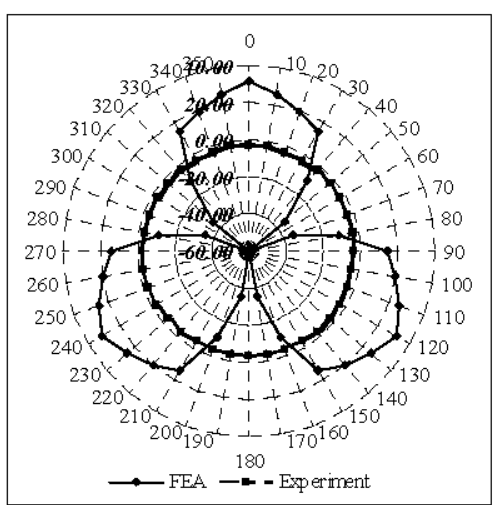

$\theta=75^{\circ}$

Fig. 4: Validation of FEA model with experimental results at $\mathrm{A}=0^{\circ}$



$\theta=0^{\circ}$

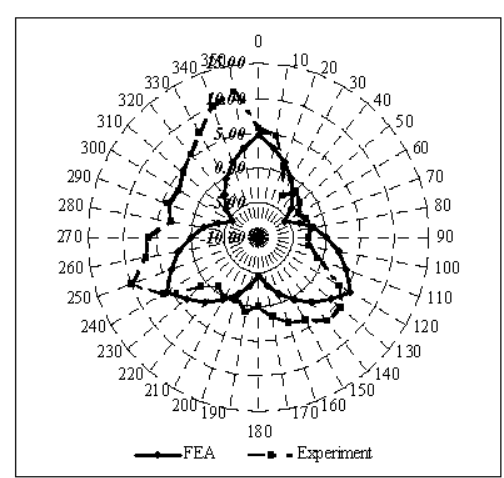

$\theta=15^{\circ}$

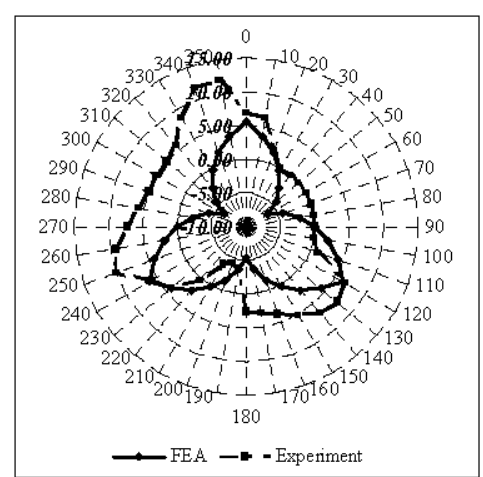

$\theta=30^{\circ}$ 


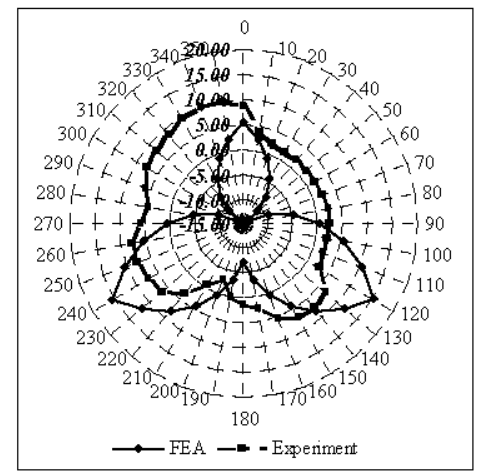

$\theta=45^{\circ}$

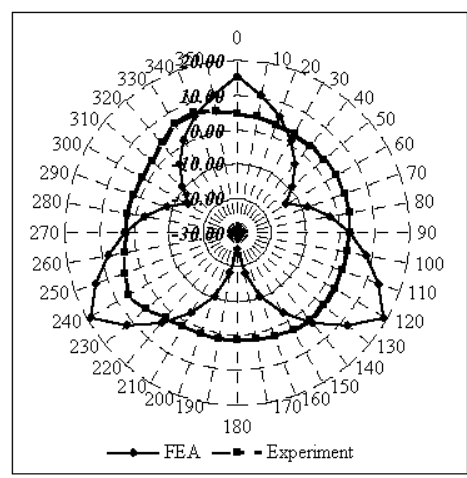

$\theta=60^{\circ}$



$\theta=75^{\circ}$

Fig 5: Validation of FEA model with experimental results at $A=45^{\circ}$

The large differences at the higher latitudes are believed to be caused by the sliding effect during the probing contact in the experiments. The sliding effect was not considered in the FEA modeling, which assumed that all contacts are perpendicular to the probe surface. Fig. 6 indicates that the probing contacts without sliding effect will significantly increase the errors at higher latitudes. According to the operating principle of the actual probe, when the probing contact is at higher latitudes, even though the contacts are made perpendicular to the surface, the sliding will always occur, which will significantly reduce the lobing or pre-travel effect. Further discussion on this can be found in [4] and [6].



Fig. 6: Probe errors at different latitudes $\left(\mathrm{A}=0^{\circ}\right)$

\section{CONCLUSIONS}

The FEA modeling results have been systematically compared and validated against the experim ental results. The agreement is generally good with shorter stylus length and probing contacts at lower latitudes near the equator of the reference sphere. The differences between the modeling and experimental results become large at higher latitudes. This is believed to be mainly caused by the sliding effects which occur during probing contact in the experiments.

The proposed methodology of using FEA for probe modeling has been successfully demonstrated. It can be used in the further studies of various probe characteristics and to improve the design and performance of CMM probes.

\section{REFERENCES}

[1] John A. Bosch, "Coordinate Measuring Machines and Systems", Marcel Dekker Inc., 1995.

[2] Q. Yang, C. Butler, P. Baird, "Error compensation of touch trigger probes", Measurement Vol. 18, No. 1, 1996, p47-57.

[3] Y. Shen, S. Moon, "Mapping of probe pretravel in dimensional measurements using neural networks computational technique" Computers in Industry 34, 1997, p295 - 306.

[4] W. Tyler Estler, et al, "Error Compensation for Touch Trigger Probes", Precision Engineering 19, 1996, p85 - 97.

[5] A. Wozniak and M Dobosz, "Metrological Feasibilities of CMM Touch Trigger Probes. Part I: 3D Theoretical Model of Probe Pre-travel", Measurement 34, pp 273-286, 2003.

[6] Y. Shen and X. Zhang, "A pre-travel model for touch trigger probes with straight styli, Part I: Vertical probes", Proceedings of North American Manufacturing Research Conference, Vol. 24, SME, Ann Arbor, Michigan, 1996, p1 27 - 132

[7] M. R. Salleh, Q. Ping Yang, Barry J., "Finite Element Modeling and Simulation of Probe System", Instrumentation and Measurement Technology Conference, 2005. IMTC 2005 Vol. 2, 16 - 19 May 2005, p849-852.

[8] M. R. Salleh, Q. Ping Yang, Barry J., "Evaluation of Touch Trigger Probe Measurement Uncertainty Using FEA" Instrumentation and Measurement Technology Conference, 2006. IMTC 2006, $24-27$ April 2006, p833-836. 